\title{
Influence of personal mobile phone ringing and usual intention to answer on driver error
}

\author{
Carol Holland and Versha Rathod
}

Psychology, School of Life \& Health Sciences, Aston University, Birmingham, B4 7ET, UK

Corresponding author: C.A. Holland, emailc.holland1@aston.ac.uk, tel +44 (0) 1212044063 , Fax: +44 (0) 1212044090 . 


\section{Abstract}

Given evidence of effects of mobile phone use on driving, and also legislation, many careful drivers refrain from answering their phones when driving. However, the distracting influence of a call on driving, even in the context of not answering, has not been examined.

Furthermore, given that not answering may be contrary to an individual's normal habits, this study examined whether distraction caused by the ignored call varies according to normal intention to answer whilst driving. That is, determining whether the effect is more than a simple matter of noise distraction. Participants were 27 young drivers (18-29 years), all regular mobile users. A Theory of Planned Behaviour questionnaire examined predictors of intention to refrain from answering calls whilst driving. Participants provided their mobile phone number and were instructed not to answer their phone if it were to ring during a driving simulation. The simulation scenario had seven hazards (e.g. car pulling out, pedestrian crossing) with three being immediately preceded by a call. Infractions (e.g. pedestrian collisions, vehicle collisions, speed exceedances) were significantly greater when distracted by call tones than with no distraction. Lower intention to ignore calls whilst driving correlated with a larger effect of distraction, as was feeling unable to control whether one answered whilst driving (Perceived Behavioural Control). The study suggests that even an ignored call can cause significantly increased infractions in simulator driving, with pedestrian collisions and speed exceedances being striking examples. Results are discussed in relation to cognitive demands of inhibiting normal behaviour and to drivers being advised to switch phones off whilst driving.

Keywords: Mobile phone distraction, driver errors, driving simulator, Theory of Planned Behaviour, younger drivers. 


\subsection{Introduction}

It is well-established that inattention when driving and improper lookout are primary causes of driving collisions (e.g., Treat, et al., 1979). For example, Hendricks, et al. (1999) reported that of 723 crashes, $37.8 \%$ were caused by driver inattention or perceptual errors. Drivers have also been shown to be at a higher risk of collisions with stationary vehicles when disrupted by a secondary task (Langham et al., 2002). One such secondary task that has been investigated is the influence of using a mobile phone whilst driving.

An increasing number of consumers own mobile phones, and mobile phone technology has progressed immensely to the extent that individuals are able to send and receive pictures, video files, and e-mail at their convenience. With increasing functionality, "on-the-go" use places a potential risk for those on the road including the driver themselves, passengers and pedestrians (Ferguson, 2003; Peters and Peters, 2002; Lam, 2002). When dialling and receiving mobile phone calls a physical interaction must be made with most units. However, the physical interaction, or amount of time with "hands off the wheel, eyes off the road" is not the only issue. The secondary task of dialling numbers, keying texts or other responses have been shown to be associated with cognitive processing demands resulting in further interference with driving performance (Haigney, et al., 2000), underlining the fundamental importance of research into the effects of mobile phone usage on driving.

On road, simulator, and accident report data have all shown a link between mobile phone use and driver errors or collisions. An on road observation study with in-car cameras (Virginia Tech Transportation Institution, 2009) reported that drivers who manually manipulate their mobile phones for calling or text messaging whilst driving were 23 times more likely to crash or be involved in an actual traffic incident. Other evidence has demonstrated that those who engage in mobile phone conversations have a higher risk of 
failing to notice traffic signals and have slower reaction times when detecting traffic signals compared to those who do not engage in mobile phone conversations (Strayer and Johnston, 2001). This is further supported by Hancock, Lesch, and Simmons (2003), finding that fewer drivers stopped for red rights in the presence of a mobile phone task than when driving without such a secondary task. Redelmeier and Tibshirani (1997) evaluated 699 mobile phone related motor vehicle collisions. From this analysis the authors reported that $24 \%$ of these individuals were found to have used their phone during a 10 minute period preceding the accident. The authors concluded that drivers using mobile phones are approximately four times more likely to be involved in a car crash than when they do not use it.

The precise effect of this kind of distraction on aspects of driving performance has also been examined. Young, et al. (2003) established that mobile phone using drivers have impaired judgments with regards to visual environments, lateral positioning and decision making skills, particularly in terms of speed of response. Additional evidence reported that drivers' braking reaction times were also shown to increase when they drove with a distraction (i.e. using a hand-held mobile phone) in comparison to driving without this distraction (Consiglio, et al., 2003) and Lamble, et al., (1999) reported that when following a lead vehicle there was an increased reaction time as well as impaired ability to maintain lane position, with increased variability in steering wheel and speed control (also Reed and Green, 1999). This accumulation of evidence indicates that specific infractions are more likely to occur when drivers use mobile phones.

However, these studies were largely investigating hand-held devices or those that require physical manipulation and diversion of visual gaze. There is also evidence to suggest that hands-free mobile usage, without physical manipulation, and other voice-activated in-car technologies, can have distraction effects on drivers' attention to the driving task or traffic 
scenarios, with McKnight and McKnight's 1993 simulation study clearly showing the separate effects of conversation on failure to respond to hazards. They found a difference between simple and complex conversations, underlining the influence of cognition. It may be that vehicle control skills (e.g. steering) would be less likely to be affected by distracting secondary tasks that involve a cognitive component only (hands-free distraction) since these are relatively well-learnt, automatic responses, as opposed to cognitively demanding decision making and response to hazards. Previous studies (e.g. Strayer, et al., 2003) have found that hands-free conversation can impair reaction time, especially in high density traffic conditions but have not compared subcomponents of the driving task. Some research has shown little difference in the effects of hands-free versus hand-held (Törnros and Bolling, 2005; 2006; Consiglio, et al., 2003), but a meta-analysis of Norwegian data, (Backer-Grøndahl and Sagberg, 2011) found that the relative risk was indeed higher for hand-held phones. One aim of the current study is to compare cognitively demanding components of the driving task with more automatic vehicle control skills.

Despite legislation against drivers' use of mobile hand-held phones in the United States, Australia and United Kingdom amongst others, (Pennay, 2008), evidence shows that bans do not have a long term affect on the drivers' behaviour without sustained reinforcement (Asari, et al., 2000; Royal, 2003), with international evidence demonstrating that many drivers' continue to engage in this behaviour even though bans have been established (e.g., Törnros \& Bolling, 2005; Pennay, 2006; McCartt et al., 2006; Svenson and Patten, 2005; Wiesenthal and Singhal, 2005). For example, Pennay (2006) showed that $43 \%$ of those who owned a mobile phone used it to answer calls when driving, $24 \%$ used their mobile phones for dialling calls, and $23 \%$ used their phone for sending and reading text messages, with only a third of these drivers using a hands-free unit. 
Nevertheless, given the widely publicised influences of mobile phone use on driving, and the legislation against mobile use in many countries, many people do avoid using their phone to make calls whilst driving or responding to calls they may receive (e.g. compare Goodman's 1999 pre-legislation figure of 85\% of people using them whilst driving with Pennay's 2006 post legislation figure of 43\%). Studies have examined the factors that may influence intention to use mobile phones in different circumstances. Several studies have used the Theory of Planned Behaviour (TPB) model (Ajzen, 1991) of predicting behavioural intention to examine this issue (e.g., Zhou et al., 2009; Walsh, et al., 2008; Rozario, et al., 2010). For example Walsh, et al. (2008) indicated that attitudes, subjective norms and perceived behavioural control (PBC) accounted for $32 \%$ of the variance in intentions to use a mobile phone whilst driving. A key benefit of such belief-based analysis allows understanding of behavioural influences and aids in identifying predictors of intentions towards a particular behaviour. Thus this information can consequently inform education and campaigns (Fishbein, 1997), and subsequently reduce the incidence of the behaviour.

Zhou et al., found that perceived behavioural control (PBC) was the variable which was indicated in regression analyses as being more important in predicting variance in behavioural intention than age, gender, or the other TPB variables of subjective norm or attitudes. The more favourable attitudes and the greater the perception of control over their ability to use a mobile phone in those situations, the more drivers' willingness to use a mobile phone increased. PBC is defined as the presence of factors that may facilitate or impede performance of the behaviour combined with one's perceived control over these factors (e.g. Ajzen, 1991). However, Zhou et al. (2009) and also Rozario et al. (2010), conceptualised PBC as ability or ease and difficulty of using a mobile phone whilst driving, whereas Walsh et al., (2008), perhaps more accurately, conceptualise it in their questions to participants as control 
over whether they use it or not whilst driving, with resultant differences in findings, Walsh et al. finding less of a role of $\mathrm{PBC}$ in predicting intention to use the phone whilst driving.

However, these studies examined intention to use a phone, not intention to refrain from using it whilst driving, and the ability to not do something, such as answering the phone, which may be a well-ingrained habit, needs separate examination. The reason for this is simply that for many people, using, or answering the phone when driving has become a habit. Habits are generally seen as more automatic responses that require less planning. Overcoming a habit, however, such as refraining from answering the phone, is more likely to be demanding of intention and control of intention (e.g. see Holland, et al., 2009 for a discussion of this issue), which is in turn likely to be demanding of attentional resources. Thus in this study, the intention to ignore the phone, or refrain from answering it, is the behaviour at issue.

Despite the prior research on the distraction effect of mobile phone use on driving, and on the effect of components of attitudes, subjective norms and perceived control on predicting intentions to perform the behaviour, the relative influence of one's normal intentions on the severity of the distraction effect of incoming mobile phone calls has not been examined. Given that many people do report that they would answer an incoming call whilst driving, the effect of ignoring one's mobile phone ring tone on driving also needs examining in the context of one's normal intentions to ignore or to answer. The research reviewed has been applied to the distraction effects of a mobile phone use (hands-free or hand-held), or to the effect of TPB variables, particularly PBC, on behavioural intentions. The role of TPB variables, particularly behavioural intention and $\mathrm{PBC}$ on the level of distraction experienced has not been investigated, with the hypothesis being that those who would normally answer their phone would find hearing their phone ring, in the context of having been instructed not to answer it, more distracting and more demanding of attentional control (inhibiting their 
normal response) than would people who would normally ignore their phone anyway. Thus, the present research aims to explore the relationships between the TPB components and ability to maintain driving performance in the context of refraining from answering a mobile phone call. The effect of this distraction, in the absence of conversation or physical phone manipulation, is assessed.

In summary, hypotheses of this study are:

1. Personal ring tones will affect measures of simulator driver behaviour in the absence of physical manipulation or response to call.

2. Such effects will be limited to cognitively demanding components of driving (response to hazards, speed control), as opposed to vehicle manipulation skills (steering).

3. Distraction effect of unanswered calls will vary according to intention to answer one's phone in normal circumstances.

To investigate the research aims, a TPB questionnaire was developed to examine whether intentions to ignore a mobile phone call tone has an effect on distractibility of driving performance, which was measured during a short driving simulator scenario task. The use of a simulator produces data with ease and has a major advantage of collecting data simultaneously with precision. The high fidelity driving simulator utilised in the present study provides precisely controlled conditions in comparison to differing methods of data collection, for example road tracks. Everyday hazards found on the road, with a distraction condition (mobile phone ringing) and no distraction condition (no mobile phone ringing), was devised in the simulation task, where responses to incidents were measured. 


\subsection{Method}

\subsection{Design and plan of analyses}

The study employed a repeated measures design with 2 levels, distraction and no distraction. All participants drove the same single simulator route, which had seven hazards, the first, third and fifth being accompanied by distraction from their own ringing mobile phone, with the remainder accompanied by no such distraction. Dependent variables were seven parameters associated with the behavioural outcome of reaction to the distractions. Data for behavioural measures were recorded by the simulator, and included the number of collisions with pedestrians, number of collisions with other vehicles, number of speeding violations (number of times $10 \%$ over the speed limit of $50 \mathrm{mph}$ ), errors in lateral deviation (i.e. number of centreline crossing and road edge excursions), off road collisions, and steering wheel deviation. Differences between driving during the period in which the hazards were visible for distraction and no distraction hazard responses for each of the dependent variables, were examined using within participants' ANOVA, except for the number of pedestrian collisions. Parametric analysis was selected as appropriate based on the fact that number of errors or steering wheel deviation in each variable made by participants were used, where, for example, two errors is twice as many as one. That is, the data were interval and thus acceptable for such analyses. Appropriate checks for normal assumptions were made. Pedestrian collision data was an exception as there was only one pedestrian in each of the distraction and no distraction conditions, and thus the data was limited to either collision or no collision. Repeated measures Chi squared was not appropriate because of the lack of pedestrian collisions in one condition, and so Wilcoxon signed ranks test was used. 
An overall total infractions variable was computed using all variables except steering wheel deviations, which was designated as a vehicle control skill. A distraction variable was then computed. This was the difference for each individual between their total number of infractions in the two conditions, representing the effect of distraction on serious errors for that individual. A regression design was then used to establish prediction of the computed distraction effect by the TPB variables for each of the behavioural measures, with the predictors: attitude, subjective norm, PBC, past behaviour, and intention (the TPB questionnaire).

\subsection{Participants}

Power analysis $($ power $=0.80$, alpha $=0.05$, and medium effect size) calculated a sample of 26 participants. The number of participants exceeded by one totalling 27. Participants were 15 female drivers and 12 male drivers aged between 18-29 years old with an average of 21.04 years $(\mathrm{SD}=6.00)$, who hold a valid driving licence, were regular drivers, and regular mobile phone users. All were Aston University students of which a few were volunteers from the psychology course receiving course credits for participation.

\subsection{Materials}

\subsubsection{Driving Simulator.}

The STISIM Drive simulator software, developed by Systems Technology Inc., was used to measure and collect participants' driving data. The simulator dashboard displayed the speedometer in miles per hour (MHP) and engine revolutions per minute (RPM) data. The software received inputs from controls consisting of a steering wheel, turn signal indicator, accelerator, brake pedal, clutch and a manual gear stick to the left of the driver's seat. This 
equipment was positioned in front of a three angled projection surface located at Aston University (shown in Figure 1). The centre projection surface was located at 1.63 meters in front of the driver's seat with two peripheral surfaces connected to the central surface at a 40 degree angle to the centre of the left and right projection surfaces. Four speakers located around the simulation and a sub-woofer behind the driver's seat represented realistic engine and road noises according to the driving scenario.

[Figure 1 inserted here]

\subsubsection{Simulation scenario.}

Throughout the simulation scenario the distance travelled was displayed in the bottom left corner of the central projection surface and gears one, two, three, four or five, depending on which gear the driver was in, was displayed in the bottom middle of the central projection surface. The scenario route was a stretch of road 6096 metres long, containing a combination of straight, gentle and sharp, right and left curves with vertical and horizontal curvature (hills). It was created in a rural setting with a single carriageway and then gradually developing into a dual carriageway with a speed limit of 50 miles per hour ( $\mathrm{mph}$, or 80 kilometres per hour, $\mathrm{km} / \mathrm{h}$ ) throughout the route. The creation of lane widths and road markings replicated real life settings including the United Kingdom's (UK's) traffic signals, speed limit road sign, 'sharp bend' road signs and 'stop' road sign. Traffic of various vehicles including vans, coaches, buses, motorcycles, different shape and colour cars and pedestrians were also included throughout the simulation. The simulation scenario included seven hazards with mobile phone call distractions at three of them, as follows:

1. Approaching vehicle at $1400 \mathrm{~m}$ (mobile phone call at $1350 \mathrm{~m}$ ). 
2. Barrels placed on the left side on the road, at $1683 \mathrm{~m}$

3. "Stop" sign intersection, at $2891 \mathrm{~m}$ (mobile phone call at $2800 \mathrm{~m}$ ).

4. Parked car pulls out onto the road when participant's vehicle is $5 \mathrm{~m}$ away from the parked car, at $4150 \mathrm{~m}$.

5. First pedestrian crossing the road at $4550 \mathrm{~m}$ (mobile phone call at $4450 \mathrm{~m}$ )

6. Intersection of traffic lights located at $4876 \mathrm{~m}$.

7. Second pedestrian crossing the road, at $4892 \mathrm{~m}$.

To ensure that both pedestrian hazards were of equal difficulty, the immediate scenario section was copied exactly into the two instances in the programming. To examine comparability between the hazards selected for distraction and those selected for no distraction, piloting drives with four participants without any distraction were assessed. There was little difference in the total number of infractions made between hazards, with few infractions apparent. The highest, a mean of 1.00 infraction occurred for the approaching vehicle hazard and the next highest was a mean of 0.5 infractions for the barrels hazard. The rest gathered means of 0.25 or zero infractions (specifically, 0.25 , or one participant for the first pedestrian, none for the second). Mobile phone distraction for the main study was paired carefully with hazards with the aim of ensuring that distraction was evenly applied across hazards that had gained more or fewer infractions in the pilot, and that hazards with distraction were spread across the drive. 


\subsubsection{TPB questionnaire}

The TPB questionnaire consisted of items about attitudes, subjective norms and PBC rated on a 7 point bipolar scale based on target behaviour (mobile phone distraction), target (mobile phone), action (distraction), time (whilst driving), and context (in different driving situations) as specified by Ajzen (1991).

Attitude was measured by taking a mean of the following statements that respondents were to complete. Statements were rated on a seven-point bipolar adjective scale: 'For me, ignoring my mobile phone when driving is:' The five pairs of adjectives were: harmful beneficial, pleasant - unpleasant, good - bad, important - not important, unsafe - safe. A measure of the reliability of the attitude statement was conducted, resulting in a low Cronbach's alpha of 0.38. Principal component analysis revealed that Item 2 (adjectives pleasant-harmful) was not correlated well with the other variables suggesting that this was measuring an affective component as opposed to an instrumental component assessed by the other adjective pairs. Leaving this item out of the reliability analysis gave a Cronbach's alpha value of .54. Ajzen (2002) recommends that it is not essential to have high internal consistency for belief based measures, as long as there is the aggregate of differing beliefs that forms an attitude. The inter-item correlation of 0.22 identifies that the aggregated variable forms a unitary component which supports aggregation (Briggs and Cheek, 1986 recommend an optimal range of the inter-item correlation .2 to .4).

The mean of three statements were used as a measure of subjective norm. Each item was rated by respondents using a seven-point bipolar scale. The three items were: 'Most people who are important to me ignore their mobile phone when driving' (completely true completely false), 'The people in my life whose opinion I value (answer - ignore) their 
mobile phone whilst driving', 'Many people like me ignore their mobile phone whilst driving' (extremely unlikely - extremely likely). A measure of the reliability of the subjective norm statements was conducted. As Cronbach's alpha values are sensitive to the number of items on a scale $(\alpha=.57)$, due to only three items on the subjective norm scale the calculation of the mean inter-item correlation was .29. This indicates reasonable internal consistency (Briggs and Cheek, 1986).

A measure of Perceived behavioural control (PBC) was obtained by calculating the mean of four items, each rated on a seven-point bipolar scale. The four items were: (i) 'For me ignoring my mobile phone whilst driving in the forthcoming month would be' (impossible - possible), (ii) It is mostly up to me whether or not I ignore my mobile phone whilst driving"; (iii) 'How much control do you believe you have over ignoring your mobile phone whilst driving' (no control - complete control), and (iv)'If I wanted to I could ignore my mobile phone whilst driving' (defiantly true - defiantly false) a measure of the reliability of the PBC statements was conducted. Cronbach's alpha was low (0.34) and principle components analysis revealed that PBC items (ii) and (iii) were not well correlated with the principle component, with (ii) possibly reflecting subjective norm characteristics. Extracting these two variables resulted in a Cronbach's alpha of 0.63 .

There were three statements measuring intention to ignore mobile phone calls whilst driving. Each statement was rated on a seven-point bipolar scale. The statements were: "I plan to ignore my mobile phone every time I drive for the next month" (strongly disagree - agree); 'I intend to ignore my mobile phone every time I drive for the next month' (extremely unlikely - extremely likely); 'I will try to ignore my mobile phone every time I drive for the next month' (defiantly true - defiantly false). A measure of the reliability of the intention statements was conducted with Cronbach's alpha $=.59$, indicating reasonable internal 
consistency and principle components analysis revealing just one component with which all three items correlated well.

Two statements assessed past behaviour on a seven-point bipolar scale: "How often have you ignored your mobile phone whilst driving in the last month?" (Never - nearly all the time); 'I have ignored my mobile phone whilst driving in the last month' (strongly agree strongly disagree). A measure of the reliability of the intention statements was conducted with Cronbach's alpha $=.82$, indicating strong internal consistency.

\subsection{Simulator Measures}

Measures were calculated on the driving simulator, number of collisions with pedestrians, number of collisions with other vehicles, number of speeding violations (number of times $10 \%$ over the speed limit of $50 \mathrm{mph}$ ), errors in lateral deviation (i.e. centreline crossing and road edge excursions), off road collisions, and steering wheel deviation/adjustments. Variables were calculated as follows from simulator data:

- Steering wheel deviation, calculated as the standard deviation of the distance from the centre (degrees, negative for left steering from the centre and positive for right steering from the centre)

- Total number of centre line crossings

- Total number of road edge excursions.

- Total number of times speed exceeded

- Collisions with other vehicles recorded as the total number of events

- Off-road collisions (e.g. with road signage) recorded as the total number of events 
- Number of collisions with pedestrians as the total number of events

\subsection{Procedure}

Prior to the commencement of data collection, ethics were approved by Aston University Ethics Committee and informed consent obtained. Participants completed a demographic questionnaire which asked for general information including gender and age only, and the TPB questionnaire. Participants were asked to provide their mobile phone number and informed it would be destroyed at the end of the simulation. Two practice trials (of the same $3500 \mathrm{~m}$ simulator drive) were given to the participants to familiarise themselves with the controls. The practice trial had one pedestrian and four intersections, of which two had traffic lights. The aim of the practice run was for participants to get used to the simulator vehicle controls, hence the use of intersections to encourage changing gear and speed. There was one pedestrian in the practice drive (at $750 \mathrm{~m}$ ), and the posted speed limit was the same as in the actual study drive (50mph). They were instructed not to drive the simulator as a game, but as they normally would when driving on the road abiding by the road signs (i.e. their normal driving behaviour). For the study drive, participants were instructed to set their mobile phone call to 'aloud' (or however their 'phone described an out loud ring tone as opposed to "silent" or "vibrate") and place their mobile phone where they normally would when driving. If their mobile phone was to go 'off' during the simulation, participants were instructed not to pick up the phone or answer the call in any way.

During the main study simulation participants' mobile phones were called three times at the following points of distance travelled 1400 meters (at approaching cars), 2900 meters (at stop sign intersection) and 4500 meters (when the first pedestrian crossed the road). 


\subsection{Results}

\subsection{Descriptive statistics}

Table 1 presents the means and standard deviations of the study's independent variables and the dependent variable of distractibility of a mobile phone call. It indicates that the average number of drivers intended not to ignore mobile phone calls. The mean rates of infractions were higher in the distraction conditions excluding collisions with other vehicles; lateral acceleration and steering wheel deviation were not higher in the distraction condition.

[Table 1 about here]

\subsection{Effect of distraction on driving performance}

In order to examine the effect of mobile phone distraction on driving performance, repeated measures ANOVAs were conducted to observe driving performance with and without the mobile phone distraction. Significant main effects of distraction were found for off road accidents, speed exceedances, and centre line crossings (see Table 1), all such that greater numbers of such infractions occurred in the context of mobile phone ring tone distraction. With the Wilcoxon signed rank test for pedestrian collisions, a significant main effect of distraction was also found. It is noteworthy that no drivers collided with pedestrians under conditions of no distraction, but $41 \%$ of the same drivers collided with the pedestrian in the distraction condition. Although the pilot study did not have enough participants for appropriate comparison, it is worth noting that this figure is also greater than the one out of the four pilot participants who collided with this pedestrian at the same point in the drive under conditions of no distraction in the small pilot. The very large effect size for speed exceedances also highlights the seriously negative effect of the mobile phone distraction. 
Given the large amount of data extracted from the simulator, measures were categorised as Infractions (total of number of off-road accidents, number of collisions with pedestrians, number of collisions with vehicles, number of speed exceedances, centreline crossings and road edge excursions); Vehicle control skills (steering wheel deviation, entered into an ANOVA as separate variables). A significant main effect of distraction was found such that more infractions occurred when distracted by mobile phone ring tones than with no ring tones $F(1,26)=59.38, p<0.01$, partial $\mathrm{y}^{2}=.0 .70$. There was no significant effect of distraction for the vehicle control skill as indicated by steering wheel deviation $[F(1,26)=.16$, $p>0.05]$.

\subsection{Relationships with TPB variables}

To investigate the relationship between intentions on the TPB measure to ignore mobile phone whilst driving and the severity of the distraction experienced by the drivers, a distraction variable was calculated for the infractions measure, consisting of infractions whilst driving with distraction (phone ringing) minus infractions whilst driving without distraction at the key hazard points indicated. The correlation between the distraction variable and intention was significant at $r(27)=0.54, \mathrm{p}<0.01$, indicating that those who had a lower intention to ignore their phone whilst driving were more distracted (performance negatively affected) by its ringing (note that a higher score on the TPB variables indicates less likely to intend to ignore mobile phone, less likely to believe significant others would ignore their mobile phone, less likely to believe it possible for them to ignore their phone whilst driving, etc).

A further correlation between the distraction effect and Perceived Behavioural Control was also demonstrated, with people who felt less in control of whether they would be able to refrain from answering their phone showing greater distraction effect $(p<0.05$, see Table 2$)$. 
[Insert Table 2 here]

\subsubsection{Effects of TPB components on intention: regression analysis}

Stepwise regression analysis was used to assess how well the TPB model plus past behaviour predicted intention to ignore mobile phone calls whilst driving. Attitudes, subjective norm and PBC were entered at Step 1 and past behaviour at Step 2. A significant model was found $[F(1,26)=9.45, p<0.01]$. The model explains $63 \%$ of the variance in intention $\left(R^{2}=.63\right)$. PBC was the only significant TPB predictor of the total amount of variance in intention to ignore phone whilst driving in the model, $t=3.23, \mathrm{p}<0.001$, with the additional variable of past behaviour also contributing, $t=2.41, p<0.05$.

3.4.2 Regression analysis predicting effect of intention, TPB predictors and past behaviour on distraction effect

Stepwise regression analysis was used to assess how well the model (intention, past behaviour and TPB variables) predicted effect of distraction. Intention was entered at Step 1, attitude, subjective norm, PBC and past behaviour were entered at Step 2. The model was significant for Step 1 only $[F(1,26)=10.44, p<0.01]$ with the total model explaining $32 \%$ of the variance $\left(R^{2}=.32\right)$. Intention was the only contributor to the model. Neither past behaviour nor TPB predictors (namely attitude, subjective norm or PBC) contributed independently to effect of distraction once the effect of intention was accounted for.

\subsection{Discussion}

Results indicated confirmation of the first hypothesis, that driving performance in the distraction condition was impaired relative to the no distraction condition, but, in line with the 
second hypothesis, this was restricted to driving infractions in that a vehicle control skill (steering wheel deviation) was not affected significantly. Regan, Lee and Young, (2008), suggested that steering wheel deviations are sensitive to visual-manual secondary task distraction, which was excluded in our method, while lane keeping (as indicated by our measure of centre-line crossing or road edge excursions) is affected by visual-manual load, but also by cognitive load such as listening to radio broadcasts (although they also cite evidence that this may actually improve under low to moderate load). The second hypothesis suggested that the actual errors (excursions) may differ from the vehicle position correction data (steering wheel deviation) in terms of the effect of distraction, and findings concur.

This implies that the risks of driving infractions overall and specifically for off road accidents, collisions with pedestrians, speed exceedances, and centre line crossing increase with the presence of a mobile phone call, even in the context of no physical manipulation of the phone and no conversation taking place. Specific types of infraction within the overall measure differed in terms of frequency, hence the benefit of collapsing across type for analysis, but it is worth noting that collisions with a pedestrian only occurred under conditions of distraction, with approximately $41 \%$ of drivers colliding with the pedestrian in the distraction condition. It may be argued that because the second pedestrian hazard was always the one without mobile phone call distraction (see methods), the lack of errors may be related to an order or practice effect. However, this explanation of the effect seems unlikely since the two identical practice drives that each participant conducted before the study drive contained a pedestrian, and so overall, the pedestrian with distraction was actually the third pedestrian participants had encountered overall. Furthermore, although the piloting of the study drive under conditions of no distraction included only four participants, only one of these collided with the pedestrian in the first instance. Speed exceedances were also frequent under distraction, implying that the 
level of speed is greatly sensitive to distractibility in comparison to the other infractions that were accounted for. Although results do confirm the hypothesis that less demanding vehicle control skills would be less affected by the distraction, and that the effect on errors in the absence of phone interaction implies cognitive demand effects, replication with eyetracking equipment could usefully check whether participants' gaze did shift while their phone was ringing, to confirm the role of "eyes off the road" as compared with cognitive demand.

Results also indicate that the third hypothesis was confirmed, that the distraction effect of unanswered calls was predicted by one's normal intention to ignore or to answer calls whilst driving. Greater intention to use a mobile phone whilst driving (to not refrain from using it) led to a greater increase in serious driver errors in the simulated drive whilst the phone was ringing. This suggests that if an individual intends not to ignore their mobile phone, a caller tone will increase the chances of an individual incurring driving infractions, resulting in poorer driving performance even if there is no physical contact with the phone. Although the effect of mobile phone tones on performance confirms previous studies demonstrating that distractibility whilst driving can be affected by noise e.g. when using a voice recognition interface (Ranney, et al., 2005) resulting in inattention, this study demonstrates clearly that this is not just an effect of noise distracting people. The new finding here is that the role of inhibiting one's normal behaviour to comply with the requirements of not answering the phone, had a further effect for those for whom refraining would not be their normal response.

Findings relative to the third hypothesis found that only PBC significantly predicted intention to ignore calls whilst driving, confirming previous TPB studies on intentions relevant to mobile phone use whilst driving (e.g. Walsh et al. 2007; Zhou et al. 2009). However, whilst these previous studies examined control or perceived ability to use a mobile 
phone whilst driving, this study examined control over ignoring its ringing and refraining from answering it. The importance of perceived behavioural control in this specific intention, and that of intention in predicting effect of distraction underlines the crucial link being made by this study, that cognitive control required to resist a normal (possibly habitual, or automatic) response is demanding of attentional control resources, and that this added demand results in driver errors. Previous work using the concept of intention to predict other behaviour variables from the health psychology literature (e.g. exercise behaviour, Hall, et al., 2008) has confirmed the relationship between self-regulatory behaviour (e.g. performing or refraining from a behaviour) and self-regulatory ability, as assessed in Hall et al.'s study using measures of executive control (a cognitive measure of ability to control the allocation of attention, for example, to inhibit irrelevant responses, e.g. Norman and Shallice, 1986). Hall et al. found that executive function strongly moderated the association between behavioural intention and actual behaviour, and we have shown that inhibiting a normal response has a significant effect on the cognitively demanding driver errors only. Previous work by Haigney, et al. (2000) concluded that even in the context of no physical interaction with a mobile phone, drivers were putting more pressure on attentional resources and this has implications for road safety as even the presence of a mobile phone call in our study seems to imply increase of the chances of serious errors and collisions.

Studies with simulators have been demonstrated as extremely useful in terms of safely assessing the influences of such distractions and being able to manipulate scenarios in a controlled manner. However, the limitation in generaliseability is recognised in that simulators cannot replicate the true nature of a driving scenario. Although a simulator provides the technology to replicate a realistic scenario with precision, the complex and intricate nature of driving on the road can not entirely be imitated in a simulator setting. For 
example, Reed and Green (1999) found that drivers' performance was poorer in the simulated environment in comparison to when on a real track, but other studies, notably with this make of simulator (STISIM), have found poorer overall performance on the road than in the simulator, but the same trends across error types and behavioural measures. For example, Schechtman et al., (2009) conducted a validation study in which they found no interactions (the same trends in any effects) in any kind of driving error, including those measured in this study, when they compared right and left turns. However, they did find significant differences in the number of anterior/posterior positioning errors, signalling errors and speed regulation errors between on road and simulator environments, with more of these errors being made on the road. Although validation studies examining comparability between simulator and on-road collisions would be unethical, Schechtman et al. did find no difference between simulator and on-road adjustment to stimuli, which included improper response to traffic or pedestrian movement. That is their data confirmed relative validity, but not necessarily absolute validity. Other studies have compared simulation with on road driving for hazard perception, arguably a more attentionally demanding task than some of the vehicle control measures in previous studies, and again, found comparability in behavioural effects - e.g. Underwood, Crundall \& Chapman, (2011) such as increased scanning and earlier eye fixations on hazards for more experienced drivers. Thus although we may expect some absolute differences in numbers of errors between simulation and on road driving, conclusions from validation studies suggest that relative differences are highly comparable, confirming the utility of high fidelity simulators such as used here for prediction of on road effects.

A further limitation of the study is that the perception of reality of driving performance may have been affected due to this simulated setting having a lack of movement. For example, although participants were instructed to drive as they normally would on the 
roads, abiding by the road signs, they did not suffer from any repercussion from their driving violations or from collisions made. However, Kass, et al., (2007) found that experienced drivers in a simulated environment committed an average of less than one infraction implying they valued the importance of instructions to drive as carefully as they would on a real road. Therefore it is important to stress such instructions to participants in order to produce valid findings which can be generalised to a real traffic setting.

A further limitation concerns participant bias when requesting participants for their personal mobile phone number immediately before the simulation task. Participants may assume they will receive a call to distract them during the simulation task, thus resulting in an unnatural reaction to the distractions. Further research could request mobile phone numbers when participants initially sign up to take part in the study to prevent such demand characteristics.

Finally, the decision to carefully fix pairings of distractions based on spread through the scenario and pilot study error levels for each hazard must be balanced against possibilities of the effects of making an error on subsequent response, e.g. to the next pedestrian, or other order effects. Further study could usefully compare both approaches.

However, this study has important implications in terms of interventions that can aid promotion in road safety. For example, educating drivers that their intentions to use a mobile phone can have an impact on the likelihood of an accident to occur. Additionally, with regards to the TPB, educating drivers that they have overall control (relating to PBC) over changing their caller setting to 'silent' or turning their phones off will reduce the chances of distractibility, thus increasing road safety. 


\subsection{Conclusion}

The present study has confirmed previous conclusions indicating that driving performance deteriorates in the presence of a mobile phone distraction, but additionally demonstrates that this occurs in the absence of interaction with the phone or conversation. The effect on the more cognitively demanding components of driving, specifically hazard avoidance and speed control, was confirmed, with more automatic level vehicle control skills not being significantly affected by personal mobile phone ring tone. Importantly, we have added significant new evidence that one's normal intention to use mobile phones whilst driving has an impact on the distraction effect and increase in serious error when a mobile phone call is heard. Whilst this research is limited to younger, less experienced drivers, results have implications for the inclusion of the role of intentions, and particularly perceived control over behaviour, in models of driver distraction. Given well established findings of reduced executive function in older adults, this study has further implications for older age groups in particular, but there are further implications for other sub-groups of drivers with reduced executive function. The findings have significant implications for road safety education and information confirming clearly that ignoring a mobile phone call whilst driving is not enough, and that switching it off or setting to silent is safer.

\section{Acknowledgments}

The Aston Driving Simulator is funded by Aston Research Centre for Healthy Ageing, ARCHA.

We acknowledge the assistance of Stephen Markham of Valentine Technologies for his invaluable advice in our simulator programming and data extraction. 


\subsection{References}

Ajzen, I., 1991. The theory of planned behaviour. Organisational behaviour and human decision processes 50, 179-211.

Ajzen, I., 2002. Constructing a TPB questionnaire: conceptual and methodological considerations. http://www.people.umass.edu/aizen/pdf/tpb.measurement.pdf.

Ansari, S., Akhdar, F., Mandoorah, M., Moutaery, K., 2000. Causes and effects of road traffic accidents in Saudi Arabia. Public Health 114(1), 37-39.

Backer-Grøndahl, A., Sagberg, F., 2011. Driving and Telephoning: Relative accident risk when using hand-held and hands-free mobile phones. Safety Science 49, 324-330

Briggs, S.R., Cheek, J.M., 1986. The role of factor analysis in the development and evaluation of personality scales. Journal of Personality 54, 106-148.

Consiglio, W., Driscoll, P., Witte, M., Berg, W.P. 2003. Effect of cellular telephone conversations and other potential interference on reaction time in a braking response. Accident Analysis and Prevention 35, 495-500.

Ferguson, S. 2003. Other high risk factors for young drivers - how graduated licensing does, doesn't, or could address them. Journal of Safety Research 34, 71-77.

Fishbein, M., 1997. Predicting, understanding, and changing socially relevant behaviours: Lessons learned. In C. McGarty, S. A. Haslam (Eds.), The message of social psychology (pp. 77-91). Cambridge: Blackwell Publishers Ltd.

Goodman, M. F., Bents, F. D., Tijerina, L., Wierwille, W., Lerner, N., Benel, D., 1999. An investigation of the safety implications of wireless communication in vehicles. Report 
summary. Retrieved 11 February 2011 from

http://www.nhtsa.dot.gov/people/injury/research/wireless/\#rep

Haigney, D.E., Taylor, R.G., and Westerman, S. J., 2000. Concurrent mobile (cellular) phone use and driving performance: task demand characteristics and compensatory processes. Transportation Research Part F 3, 113-121.

Hall, P.A., Fong, G.T., Epp, L.J., Elias, L.J., 2008. Executive function moderates the intention-behaviour link for physical activity and dietary behaviour. Psychology and Health 23, 309-326.

Hancock, P.A., Lesch, M.F., Simmons, L., 2003. The distraction effects of phone use during a crucial driving manoeuvre. Accident Analysis and Prevention 35, 501-514.

Hendricks, D. L., Fell, J. C., Freedman, M., 1999. The relative frequency of unsafe driving acts in serious traffic crashes. Retrieved 11 February 2011 from http://www.nhtsa.dot.gov/people/injury/research/UDAshortrpt/index.html

Holland, C.A., Hill, R., Cooke, R., 2009. Understanding the role of self-identity in habitual risky behaviours: pedestrian road crossing decisions across the lifespan. Health Education Research 24, 674-685

Kass, S.J., Cole, K.S., Stanny, C.J., 2007. Effects of distraction and experience on situation awareness and simulated driving. Transportation Research Part F, 10, 321-329.

Lam, L.T., 2002. Distractions and the risk of car-crash injury: The effect of driver's age. Journal of Safety Research 33, 411-419. 
Lamble, D., Kauranen, T., Laasko, M., Summala, H., 1999. Cognitive load and detection thresholds in car following situations: safety implications for using mobile (cellular) telephones while driving. Accident Analysis and Prevention 31, 617-623.

Langham, M., Hole, G., Edwards, J., O’Neil, C., 2002. An analysis of 'looked but failed to see' accidents involving parked police cars. Ergonomics 45, 167-185.

McCartt, A.T., Hellinga, L.A., Bratiman, K.A., 2006. Cell phones and driving: review of research. Traffic Injury Prevention 7, 89-106.

McKnight, A.J. \& McKnight, A.S. (1993) The effect of cellular phone use upon driver attention, Accident Analysis and Prevention 25, 259-265

Norman, D. Shallice, T. 1986. Attention to action: Willed and automatic control of behavior. In R. Davidson, G. Schwartz, D. Shapiro (Eds), Consciousness and self regulation: Advances in research and theory (Vol. 4, pp. 1-18). New York, NY: Plenum.

Pennay, D., 2006. Community attitudes to road safety: Community attitudes survey wave 18, 2005 (No. CR 227). Australian Transport Safety Bureau, Canberra.

Pennay, D., 2008. Community attitudes to road safety: Community attitudes survey wave 20, Road Safety Report 3. Australian Transport Safety Bureau: Canberra.

Peters, G.A., Peters, B.J., 2002. The distracted driver. Journal of the Royal Society for the Promotion of Health 121, 23-28.

Ranney, T.A., Harbluk, J.L., Noy, Y.I., 2005. Effects of voice technology on test track driving performance: Implications for driver distraction, Human Factors 47. 439-454.

Redelmeier, D.A., Tibshirani, R.J., 1997. Association between cellular-telephone calls and motor vehicle collisions. The New England Journal of Medicine 336, 453-458. 
Reed, M.P., Green, P.A., 1999. Comparison of driving performance on-road and in a low-cost simulator using a concurrent telephone dialling task. Ergonomics 42, 1015-1037.

Regan, M.A., Lee, J.D. Young, K.L. 2008 Driver distraction: theory, effects, and mitigation. Boca Raton, CRC Press.

Royal, D., 2003. Volume 1: Findings. National survey of distracted and drowsy driving attitudes and behaviours, 2002. National Highway Traffic Safety Administration (NHTSA) Report Number: DOT HS 809566. Retrieved 1 March 2011 from http://www.nhtsa.dot.gov/people/injury/drowsy_driving1/surveydistractive03/index.htm.

Rozario, M., Lewis, I., White, K.M., 2010. An examination of the factors that influence drivers' willingness to use hand-held mobile phones. Transportation Research Part F $13,365-376$.

Shechtman, O., Classen, S., Awadzi, K., Mann, W. 2009. Comparison of driving errors between on-the-road and simulated driving assessment: A validation study. Traffic Injury Prevention 10, 379-385

Strayer, D. L., Johnston, W.A., 2001. Driven to distraction: Dual-task studies of simulated driving and conversing on a cellular phone. Psychological Science 12, 462-466.

Strayer, D.L., Drews, F.A., Johnston, W.A., 2003. Cell phone induced failures of visual attention during simulated driving. Journal of Experimental Psychology: Applied 9, $23-32$. 
Svenson, O., \& Patten, C.J.D., 2005. Mobile phones and driving: a review of contemporary research. Cognition, Technology and Work 7, 182-197.

Törnros, J.E.B., Bolling, A.K. 2005. Mobile phone use - Effects of handheld and hands-free phones on driving performance. Accident Analysis and Prevention 37, 902-909.

Törnros, J.E.B., Bolling, A., 2006. Mobile phone use - effects of conversation on mental workload and driving speed in rural and urban environments. Transportation Research Part F: Traffic Psychology and Behaviour 9, 298-306.

Treat, J., Tumbas, N., McDonald, S., Shinar, D., Hume, R., Mayer, R., Stansifer, R., Castallan, N., 1979. Tri-level study of the causes of traffic accidents: Executive summary. Bloomington: University of Indiana. (NTIS No. DOT HS-805 099).

Underwood, G., Crundall, D., Chapman, P., 2011. Driving simulator validation with hazard perception. Transport Research Part F: Traffic Psychology and Behaviour 14, 435446.

Virginia Tech Transportation Institution, 2009. New data from VTTI provides insight into cell phone use and driving distraction. Retrieved 17 September 2010 from $<$ http://www.vtti.vt.edu/PDF/7-22-09-VTTIPress_Release_Cell_phones_and_Driver_Distraction.pdf $>$

Walsh, S.P., White, K.M., Hyde, M.K., Watson, B.C., 2008. Dialling and driving: Factors influencing intentions to use a mobile phone while driving. Accident Analysis and Prevention 40, 1893-1900. 
Wiesenthal, D.L., Singhal, D., 2005. Is it safe to use a cellular telephone while driving? In: Hennessy, D.A., Wiesenthal, D.L. (Eds.), Contemporary Issues in Road User Behaviour and Traffic Safety. (pp. 227-244). Nova Science Publishers: New York.

Young, K., Regan, M. \& Hammer, M., 2003 Driver distraction: a review of the literature, Monash University Accident Research Centre, Report No. 206

Zhou, R. Wu, C., Rau, P.L.P., Zhang, W., 2009. Young driving learners' intention to use a handheld or hands-free mobile phone when driving. Transportation Research Part F $12,208-217$. 
Figure Captions

Figure 1: The Aston Driving Simulator set up

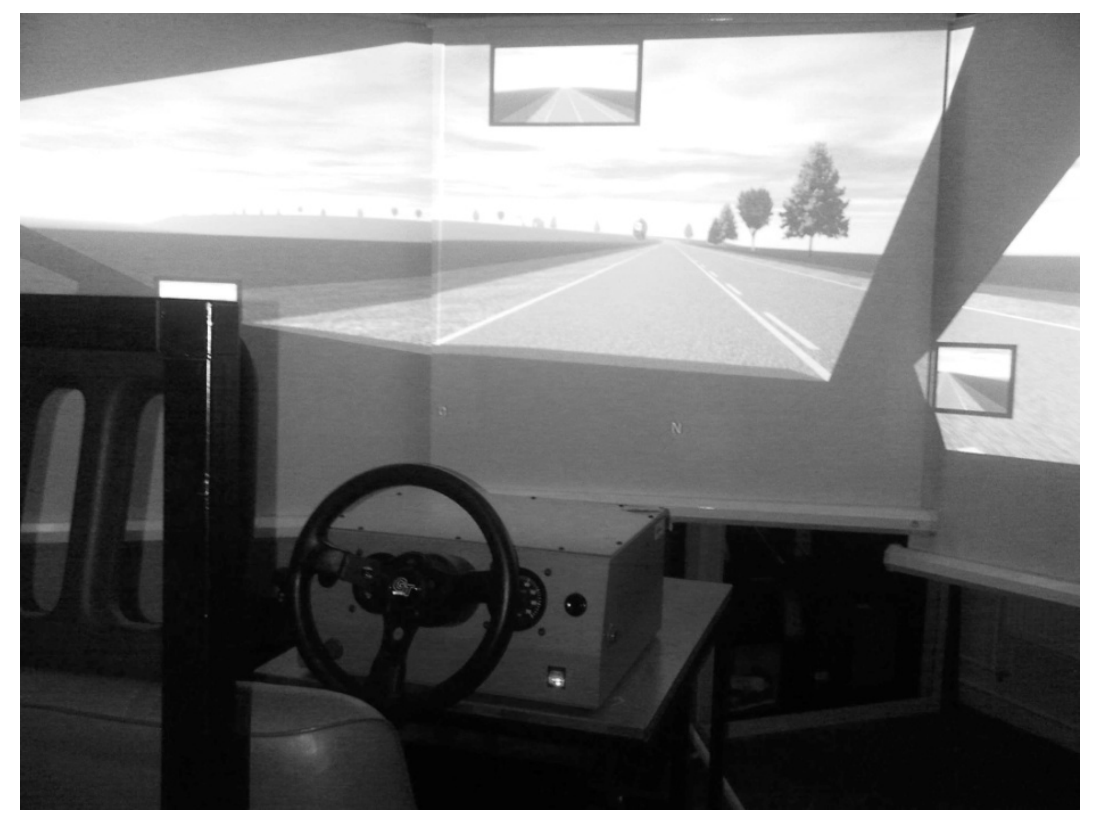


Table 1

Descriptive statistics of TPB measures and performance in the distraction and no distraction conditions.

\begin{tabular}{|c|c|c|c|c|}
\hline Variables & $\begin{array}{l}\text { TPB } \\
\text { variables }\end{array}$ & $\begin{array}{l}\text { Distraction } \\
\text { condition } \\
\text { (mobile phone } \\
\text { call) }\end{array}$ & $\begin{array}{l}\text { No distraction } \\
\text { condition } \\
\text { (no mobile } \\
\text { phone call) }\end{array}$ & $\begin{array}{l}\text { Significance of } \\
\text { Difference }\end{array}$ \\
\hline Intention $^{\mathrm{a}}$ & $5.62(1.11)$ & & & \\
\hline Attitude $^{\mathrm{a}}$ & $1.43(1.13)$ & & & \\
\hline $\mathrm{SN}^{\mathrm{a}}$ & $3.95(0.87)$ & & & \\
\hline $\mathrm{PBC}^{\mathrm{a}}$ & $5.79(0.81)$ & & & \\
\hline Past behaviour ${ }^{\mathrm{a}}$ & $4.96(1.59)$ & & & \\
\hline $\begin{array}{l}\text { Steering wheel } \\
\text { deviation }^{\mathrm{C}}\end{array}$ & & $9.17(4.78)$ & $9.56(1.48)$ & $\mathrm{F}(1,26)=0.16, \mathrm{NS}$ \\
\hline Off road accident ${ }^{\mathrm{I}}$ & & $0.15(0.36)$ & $0(0)$ & $\begin{array}{l}\mathrm{F}(1,26)=4.52 \\
\mathrm{p}<0.05\end{array}$ \\
\hline $\begin{array}{l}\text { Collisions with } \\
\text { other vehicles }^{\mathrm{I}}\end{array}$ & & $0(0)$ & $0(0)$ & \\
\hline $\begin{array}{l}\text { Collisions with } \\
\text { pedestrians }^{I}\end{array}$ & & $0.41(0.50)$ & $0(0)$ & $\mathrm{Z}=-3.32, \mathrm{p}<0.001$ \\
\hline Speed & & $4.19(2.47)$ & $1.37(1.18)$ & $F(1,26)=52.44$ \\
\hline exceedances $^{\mathrm{I}}$ & & & & $\mathrm{p}<0.001$ \\
\hline
\end{tabular}




\begin{tabular}{llll}
\hline Centre line & $0.67(0.78)$ & $0.07(0.27)$ & $\mathrm{F}(1,26)=16.98$, \\
crossing $^{\mathrm{I}}$ & & & $\mathrm{p}<0.001$ \\
Road edge $^{\text {excursions }}{ }^{\mathrm{I}}$ & $1.11(0.97)$ & $0.93(1.00)$ & $\mathrm{F}(1,26)=0.52, \mathrm{NS}$ \\
\end{tabular}

The values are given as mean (S.D) SN: Subjective norm; PBC: Perceived behavioural control;

${ }^{\text {a }}$ Scales ranged from (1) more likely to ignore mobile phone call (7) least likely to ignore mobile phone call

${ }^{\mathrm{I}}$ Infraction; ${ }^{\mathrm{C}}$ Control skill. 


\section{Table 2}

Pearson correlation coefficient of TPB variables, past behaviour and distraction effect.

\begin{tabular}{llllll}
\hline Variable & Intention & Attitude & PBC & SN & Past \\
& & & & & behaviour \\
\hline Intention & - & - & - & - & - \\
Attitude & .18 & - & - & - & - \\
PBC & $.69 * *$ & .21 & - & - & \\
SN & $.52^{* *}$ & -.09 & $.47^{*}$ & - & \\
Past & $.76^{* *}$ & .22 & $.68^{* *}$ & .63 & - \\
behaviour & & & & & \\
Distraction & $.54 * *$ & .19 & $.39 *$ & .18 & .35 \\
effect & & & & & \\
\hline$* p<0.05 ; * p<0.01$. & & & & &
\end{tabular}

